\title{
Blackbody Radiation in the Rectangular Wave Guide
}

\author{
W. Eckhardt \\ Abteilung für Theoretische Physik Universität Ulm, D-7900 Ulm
}

Z. Naturfosch. 34a, 292-299 (1979), received December 16, 1978

\begin{abstract}
Electromagnetic (EM-)fluctuations in the rectangular wave guide are considered. In the limit of the empty (lossless) wave guide the partial mode densities, the volume averaged correlations and the internal energy are discussed in both, the high and the low temperature representation. The results are compared with the corresponding quantities in the cube, the slab resonator and the infinite isotropic space.
\end{abstract}

\section{Introduction}

In the last years numerous articles concerning the influence of boundaries on the properties of the thermal radiation field (blackbody radiation) have been published [1-16].

Due to the boundaries or due to the finite volume occupied by the field the correlations of the EMfield are changed compared to those in the infinite space $[17-22]$.

In this paper we will consider the hitherto unknown blackbody radiation field in the rectangular wave guide with perfectly conducting walls. We assume that the wave guide is homogeneously filled with an isotropic dielectric medium $(\varepsilon=$ $\varepsilon^{\prime}+\varepsilon^{\prime \prime}$, magnetic permeability $\mu=1$ ).

The dissipative properties of the medium are directly connected with spontaneous fluctuations of charges and currents leading to a randomly fluctuating polarisation field $(\boldsymbol{P}(\boldsymbol{r}, t))$. The thermal radiation field is a consequence of this polarisation field.

If the quantity $\hat{A}_{i}(\boldsymbol{r}, t)$ denotes the space and time dependent operator of one of the components of the electric $(\boldsymbol{E})$, magnetic $(\boldsymbol{M})$, polarisation $(\boldsymbol{P})$ or magnetisation $(\boldsymbol{M})$ field, the corresponding symmetrized correlation function can be written in the form:

$$
A_{i j}^{(\mathrm{s})}\left(\boldsymbol{r}, \boldsymbol{r}^{\prime}, t, t^{\prime}\right)=\left\langle\frac{1}{2}\left\{A_{i}(\boldsymbol{r}, t), A_{j}\left(\boldsymbol{r}^{\prime}, t^{\prime}\right)\right\}\right\rangle .
$$

The angular brackets denote statistical average with respect to the density operator

$$
\hat{\varrho} \sim \exp \left(-\hat{H} / k_{\mathrm{B}} T\right),
$$

where $\hat{H}$ represents the Hamiltonian of the system. The curly brackets are anticommutator brackets.

Reprint requests to W. Eckhardt. - Please order a reprint rather than making your own copy.

$0340-4811 / 79 / 0300-0292 \$ 01.00 / 0$
The Fourier transformed quantity of (1.1) is defined by the relation

$$
\begin{aligned}
& A_{i j}^{(\mathrm{s})}\left(\boldsymbol{r}, \boldsymbol{r}^{\prime}, t, t^{\prime}\right) \\
& =\int_{-\infty}^{\infty} \frac{\mathrm{d} \omega}{2 \pi} \int_{-\infty}^{\infty} \frac{\mathrm{d} \omega^{\prime}}{2 \pi} e^{-i \omega t} e^{-i \omega^{\prime} t^{\prime}} A_{i j}^{(\mathrm{s})}\left(\boldsymbol{r}, \boldsymbol{r}^{\prime}, \omega, \omega^{\prime}\right) .
\end{aligned}
$$

Due to the stationarity of the process the WienerKhinchine theorem is valid:

$$
\begin{aligned}
A_{i j}^{(\mathrm{s})}\left(\boldsymbol{r}, \boldsymbol{r}^{\prime}\right. & \left., \omega, \omega^{\prime}\right) \\
& =\left(A_{i}(\boldsymbol{r}) A_{j}\left(\boldsymbol{r}^{\prime}\right)\right)_{\omega}^{(\mathrm{s})} \delta\left(\omega+\omega^{\prime}\right) .
\end{aligned}
$$

Therefore (1.1) only depends on $t-t^{\prime}=\tau$. The correlations of the polarisation field are determined by the fluctuation dissipation theorem (FDT) [22]:

$$
\begin{aligned}
& P_{i j}^{(\mathrm{s})}\left(\boldsymbol{r}, \boldsymbol{r}^{\prime}, \omega, \omega^{\prime}\right)=\left\langle\frac{1}{2}\left\{P_{i}(\boldsymbol{r}, \omega), P_{j}\left(\boldsymbol{r}^{\prime}, \omega^{\prime}\right)\right\}\right\rangle \\
& =\hbar \operatorname{coth} \frac{\hbar \omega}{2 k_{\mathrm{B}} T} \frac{1}{4 \pi} \varepsilon^{\prime \prime}(\omega) \delta_{i j} \delta\left(\boldsymbol{r}-\boldsymbol{r}^{\prime}\right) \delta\left(\omega+\omega^{\prime}\right) .
\end{aligned}
$$

The correlations of the magnetic moments vanish $\left(\mu^{\prime \prime}=0\right)$. Equation (1.4) expresses the connection between the dissipative properties of the medium and the fluctuations of the random forces. Equation (1.4) is independent of the shape and the boundary of the medium.

Our procedure will be as follows: We have to solve the Maxwell equations in the rectangular wave guide and we obtain the $\boldsymbol{E}$ - and $\boldsymbol{H}$-field as functionals of the $\boldsymbol{P}$ - and $\boldsymbol{M}$-field. Then, the bilinear quantities in $\boldsymbol{E}$ and $\boldsymbol{H}$ are obtained with the aid of Equation (1.4).

We will compare our results with the corresponding quantities in the cube $[2,3]$ and in the slab geometry $[6,13]$.

\section{Response Functions and Correlation Tensors}

In this section we follow the procedure which was used in Ref. [15]. We put the origin of the coordinate 
system on one edge of the wave guide so that the cross section is described by the coordinates $0 \leqq x \leqq L_{1}$ and $0 \leqq y \leqq L_{2}$. The $z$-axis is perpendicular to the cross section.

We expand the $\boldsymbol{E}, \boldsymbol{H}, \boldsymbol{P}$ and $\boldsymbol{M}$ field in terms of orthonormal and complete sets of functions which fulfil the appropriate boundary conditions (the tangential components of the $\boldsymbol{E}$ and $\boldsymbol{P}$ and the normal componants of the $\boldsymbol{H}$ and $\boldsymbol{M}$ fields vanish on the boundary.):

$$
\begin{aligned}
\boldsymbol{\Phi}_{\boldsymbol{k}}(\boldsymbol{r}) & =N_{\boldsymbol{k}}\left(\cos k_{1} x \sin k_{2} y, \sin k_{1} x \cos k_{2} y, \sin k_{1} x \sin k_{2} y\right)(2 \pi)^{-1 / 2} e^{i k_{3} z}=\boldsymbol{\Phi}_{\boldsymbol{k}_{\|}}(x, y)(2 \pi)^{-1 / 2} e^{i k_{3} z}, \\
\boldsymbol{\Psi}_{\boldsymbol{k}}(\boldsymbol{r}) & =N_{\boldsymbol{k}}\left(\sin k_{1} x \cos k_{2} y, \cos k_{1} x \sin k_{2} y, \cos k_{1} x \cos k_{2} y\right)(2 \pi)^{-1 / 2} e^{i k_{3} z}=\Psi_{\boldsymbol{k}_{\|}}(x, y)(2 \pi)^{-1 / 2} e^{i k_{3} z} . \\
k_{1} & =n_{1} \pi / L_{1} \text { and } k_{2}=n_{2} \pi / L_{2} ; n_{1}, n_{2}=0,1,2,3, \ldots ; \\
\boldsymbol{k}_{\|} & =\left(k_{1}, k_{2}\right), k_{\|}=\left|\boldsymbol{k}_{\|}\right|, k_{3} \text { varies continuously from }-\infty \text { to }+\infty ; \\
N_{\boldsymbol{k}} & =2\left(L_{1} L_{2}\right)^{-1 / 2} \text { for } n_{1}, n_{2} \neq \mathbf{0}, N_{\boldsymbol{k}}=\left(2 / L_{1}\right)^{1 / 2} \text { if } n_{2}=0 \text { and } N_{\boldsymbol{k}}=\left(2 / L_{2}\right)^{1 / 2} \text { if } n_{1}=0 .
\end{aligned}
$$

We insert these expansions in the Maxwell equations and obtain linear relations among the $\boldsymbol{k}$ dependent expansion coefficients of the fields.

In $\boldsymbol{k}$-space Eq. (1.4) can be written as follows:

$$
\left(P_{i}(\boldsymbol{k}) P_{j}\left(\boldsymbol{k}^{\prime}\right)\right)_{\omega}^{(\mathrm{s})}=\hbar \operatorname{coth} \frac{\hbar \omega}{2 k_{\mathrm{B}} T}(4 \pi)^{-1} \delta_{i j} \varepsilon^{\prime \prime}(\omega) \delta_{k_{1}, k_{1}} \delta_{k_{2}, k_{2}} \delta\left(k_{3}+k_{3^{\prime}}\right) .
$$

Equation (2.3) leads to the correlation of the $\boldsymbol{E}$ and $\boldsymbol{H}$ field in $\boldsymbol{k}$ space and a straightforward calculation including an integration over $k_{3}$, yields the symmetrized spectra of the correlations in $\boldsymbol{r}$-space:

$$
\begin{aligned}
& \left(E_{i}(\boldsymbol{r}) E_{j}\left(\boldsymbol{r}^{\prime}\right)\right)_{\omega}^{(\mathrm{s})}=E_{i j}^{(\mathrm{s})}\left(\boldsymbol{r}, \boldsymbol{r}^{\prime}, \omega\right)=\hbar \operatorname{coth} \frac{\hbar \omega}{2 k_{\mathrm{B}} T} \operatorname{Im} \chi_{i j E E}\left(\boldsymbol{r}, \boldsymbol{r}^{\prime}, \omega\right), \\
& \left(H_{i}(\boldsymbol{r}) H_{j}\left(\boldsymbol{r}^{\prime}\right)\right)_{\omega}^{(\mathrm{s})}=H_{i j}^{(\mathrm{s})}\left(\boldsymbol{r}, \boldsymbol{r}^{\prime}, \omega\right)=\hbar \operatorname{coth} \frac{\hbar \omega}{2 k_{\mathrm{B}} T} \operatorname{Im} \chi_{i j H H}\left(\boldsymbol{r}, \boldsymbol{r}^{\prime}, \omega\right), \\
& \left(E_{i}(\boldsymbol{r}) H_{j}\left(\boldsymbol{r}^{\prime}\right)\right)_{\omega}^{(\mathrm{s})}=G_{i j}\left(\boldsymbol{r}, \boldsymbol{r}^{\prime}, \omega\right)=\hbar \operatorname{coth} \frac{\hbar \omega}{2 k_{\mathrm{B}} T}\left(-i \operatorname{Re} \chi_{i j E H}\left(\boldsymbol{r}, \boldsymbol{r}^{\prime}, \omega\right)\right) .
\end{aligned}
$$

In (2.4)-(2.6) we defined the response functions:

$$
\begin{aligned}
& \operatorname{Im} \chi_{i j E E}\left(\boldsymbol{r}, \boldsymbol{r}^{\prime}, \omega\right)=\frac{2 \pi \omega|\omega|}{c \sqrt{\varepsilon^{\prime}}} \sum_{n_{1}, n_{2}=0}^{\infty}\left(\frac{\omega^{2}}{c^{2}} \varepsilon^{\prime}-k_{\|^{2}}\right)^{-1 / 2} \theta\left(\frac{\omega^{2}}{c^{2}} \varepsilon^{\prime}-k_{\|^{2}}\right) \\
& \cdot\left[\delta_{i j}-\frac{c^{2}}{\omega^{2} \varepsilon^{\prime}} \widetilde{k_{i}} \widehat{k}_{j}\right] \Phi_{\boldsymbol{k}_{\|}}^{i}(x, y) \Phi_{\boldsymbol{k}_{\|}}^{j}\left(x^{\prime}, y^{\prime}\right) \cos \left[\left(z-z^{\prime}\right)\left(\frac{\omega^{2}}{c^{2}} \varepsilon^{\prime}-k_{\|^{2}}\right)^{1 / 2}\right] \text {, } \\
& \operatorname{Im} \chi_{i j H H}\left(\boldsymbol{r}, \boldsymbol{r}^{\prime}, \omega\right)=\frac{2 \pi \omega|\omega|}{c \sqrt{\varepsilon^{\prime}}} \sum_{n_{1}, n_{2}=0}^{\infty}\left(\frac{\omega^{2}}{c^{2}} \varepsilon^{\prime}-k_{\|^{2}}\right)^{-1 / 2} \theta\left(\frac{\omega^{2}}{c^{2}} \varepsilon^{\prime}-k_{\|^{2}}\right) \\
& \cdot\left[\delta_{i j}-\frac{c^{2}}{\omega^{2} \varepsilon^{\prime}} \overline{k_{i}} \bar{k}_{j}\right] \Psi_{\boldsymbol{k}_{\|}}^{i}(x, y) \Psi_{\boldsymbol{k}_{\|}}^{j}\left(x^{\prime}, y^{\prime}\right) \cos \left[\left(z-z^{\prime}\right)\left(\frac{\omega^{2}}{c^{2}} \varepsilon^{\prime}-k_{\|}\right)^{1 / 2}\right], \\
& \operatorname{Re} \chi_{i j E H}\left(\boldsymbol{r}, \boldsymbol{r}^{\prime}, \omega\right)=\frac{2 \pi|\omega|}{\sqrt{\varepsilon^{\prime}}} \sum_{n_{1}, n_{2}=0}^{\infty}\left(\frac{\omega^{2}}{c^{2}} \varepsilon^{\prime}-k_{\|^{2}}\right)^{-1 / 2} \theta\left(\frac{\omega^{2}}{c^{2}} \varepsilon^{\prime}-k_{\|^{2}}\right) \\
& \cdot \varepsilon_{i j l} \tilde{k}_{l} \Phi_{\boldsymbol{k}_{\|}}^{i}(x, y) \Psi_{k_{\|}}^{j}\left(x^{\prime}, y^{\prime}\right) \cos \left[\left(z-z^{\prime}\right)\left(\frac{\omega^{2}}{c^{2}} \varepsilon^{\prime}-k_{\|}^{2}\right)^{1 / 2}\right] \text {. }
\end{aligned}
$$

In (2.7) $-(2.9)$ we already have taken the limit $\varepsilon^{\prime \prime} \rightarrow 0 . \theta$ denotes the Heaviside step function: $\theta(\eta)=0$ if $\eta \leqq 0$ and $\theta(\eta)=1$ if $\eta>0, \varepsilon_{i j l}$ is the complete antisymmetric tensor of Levi Civita. The symbols $\widehat{k_{i} k_{j}}$ and $\tilde{k}_{l}$ denote operators with the properties 


$$
\widehat{k_{i}} \widehat{k_{j}}=\left\{\begin{array}{rcc}
\frac{\partial}{\partial z} k_{j} & \text { for } & j=1,2 \\
-k_{i} \frac{\partial}{\partial z} & \text { for } & i=1,2, \\
k_{i} k_{j} & \text { for } & i, j \neq 3, \\
-\frac{\partial^{2}}{\partial z^{2}} & \text { for } & i=j=3
\end{array}\right.
$$

and

$$
\tilde{k}_{l}=\left\{\begin{array}{ccc}
-\frac{\partial}{\partial z} & \text { for } & l=3, \\
k_{l} & \text { for } & l=1,2 .
\end{array}\right.
$$

The response functions depend explicitely on $x, y$ and $x^{\prime}, y^{\prime}$. They are translationally invariant with respect to the $z$-direction. (For comparison, see the response functions $(5.2)-(5.4)$ in [15].)

If $\omega^{2} \varepsilon^{\prime} / c^{2}=k_{\|}{ }^{2}$ i.e. $k_{3}=0$ there are poles in $(2.7)-(2.9)$, which lead to singularities in the partial mode densities and the density of states. The normal ordered correlations which are closely related to measurable quantities in photon detection experiments can be obtained from the symmetrized versions [7]

$$
\begin{aligned}
& E_{i j}^{(N)}\left(\boldsymbol{r}, \boldsymbol{r}^{\prime}, \tau\right)=\int_{0}^{\infty} \frac{\mathrm{d} \omega}{2 \pi} 2 \hbar e^{i \omega \tau}\left[\exp \frac{\hbar \omega}{k_{\mathrm{B}} T}-1\right]^{-1} \operatorname{Im} \chi_{i j E E}\left(\boldsymbol{r}, \boldsymbol{r}^{\prime}, \omega\right)=\int_{0}^{\infty} \frac{\mathrm{d} \omega}{2 \pi} E_{i j}^{(N)}\left(\boldsymbol{r}, \boldsymbol{r}^{\prime}, \omega\right) e^{i \omega \tau}, \\
& H_{i j}^{(N)}\left(\boldsymbol{r}, \boldsymbol{r}^{\prime}, \tau\right)=\int_{0}^{\infty} \frac{\mathrm{d} \omega}{2 \pi} 2 \hbar e^{i \omega \tau}\left[\exp \frac{\hbar \omega}{k_{\mathrm{B}} T}-1\right]^{-1} \operatorname{Im} \chi_{i j H H}\left(\boldsymbol{r}, \boldsymbol{r}^{\prime}, \omega\right)=\int_{0}^{\infty} \frac{\mathrm{d} \omega}{2 \pi} H_{i j}^{(N)}\left(\boldsymbol{r}, \boldsymbol{r}^{\prime}, \omega\right) e^{i \omega \tau}, \\
& G_{i j}^{(N)}\left(\boldsymbol{r}, \boldsymbol{r}^{\prime}, \tau\right)=\int_{0}^{\infty} \frac{\mathrm{d} \omega}{2 \pi} 2 \hbar e^{i \omega \tau}\left[\exp \frac{\hbar \omega}{k_{\mathrm{B}} T}-1\right]^{-1} i \operatorname{Re} \chi_{i j E H}\left(\boldsymbol{r}, \boldsymbol{r}^{\prime}, \omega\right)=\int_{0}^{\infty} \frac{\mathrm{d} \omega}{2 \pi} G_{i j}^{(N)}\left(\boldsymbol{r}, \boldsymbol{r}^{\prime}, \omega\right) e^{i \omega \tau} .
\end{aligned}
$$

The last terms in (2.12)-(2.14) define the spectra of the normal ordered correlations with respect to the positive frequency range.

\section{Partial Mode Densities}

We define the volume averaged correlations

$$
\begin{aligned}
& \Gamma_{E}^{i}(\tau)=\int_{v} E_{i i}^{(N)}(\boldsymbol{r}, \boldsymbol{r}, \tau) \mathrm{d}^{3} r, \\
& \Gamma_{H}^{i}(\tau)=\int_{v} H_{i i}^{(N)}(\boldsymbol{r}, \boldsymbol{r}, \tau) \mathrm{d}^{3} r .
\end{aligned}
$$

Eqs. (3.1) and (3.2) can be written in the form

$$
\begin{aligned}
\Gamma_{E}^{i}(\tau)= & \int_{0}^{\infty} \hbar \omega e^{i \omega \tau} \\
& \cdot\left[\exp \left(\frac{\hbar \omega}{k_{\mathrm{B}} T}\right)-1\right]^{-1} 2 \pi D_{E}^{i}(\omega), \\
\Gamma_{H}^{i}(\tau)= & \int_{0}^{\infty} \hbar \omega e^{i \omega \tau} \\
& \cdot\left[\exp \left(\frac{\hbar \omega}{k_{\mathrm{B}} T}\right)-1\right]^{-1} 2 \pi D_{H}^{i}(\omega) .
\end{aligned}
$$

In (3.3) and (3.4) we have introduced the partial mode densities $D_{E}^{i}(\omega)$ and $D_{H}^{i}(\omega)$ [14].

The summation of the partial mode densities leads to the usual mode density or density of states $D(\omega)$ which is relevant for the calculation of the global thermodynamic properties of the blackbody radiation:

$$
D(\omega)=\frac{1}{2} \sum_{i=1}^{3}\left[D_{E}^{i}(\omega)+D_{H}^{i}(\omega)\right] .
$$

For the cube as well as for the isotropic infinite space all partial mode densities are equal $\left(D_{E}^{i}(\omega)=\right.$ $\left.D_{H}^{i}(\omega)=\frac{1}{3} D(\omega)\right)$. This is, however, no longer valid in our system and in the slab geometry [14]. The integration in (3.1) and (3.2) extends over the infinite $z$-axis and therefore we actually have to consider the quantities

$$
\lim _{L_{3} \rightarrow \infty} \frac{1}{V} \Gamma_{\{H\}} \text { and } \lim _{L_{3} \rightarrow \infty} \frac{1}{V} D_{\{H\}}^{i}\left(V=L_{1} L_{2} L_{3}\right)
$$

which yield finite values. 
We introduce the quantities

$$
\tilde{\boldsymbol{n}}=\left(\tilde{n}_{1}, \tilde{n}_{2}\right)=\left(\left(L_{2} / L_{1}\right)^{1 / 2} n_{1},\left(L_{1} / L_{2}\right)^{1 / 2} n_{2}\right), \quad|\tilde{\boldsymbol{n}}|=\tilde{n},
$$

and

$$
\omega_{0}=\pi c\left(L_{1} L_{2}\right)^{-1 / 2} .
$$

Inserting (2.7) and (2.8) in (2.12) and (2.13) we find in the limit $\varepsilon \rightarrow 1$ :

$$
\begin{aligned}
& D_{E}^{x}(\omega) / V=\frac{1}{\omega\left(L_{1} L_{2}\right)^{3 / 2}} \sum_{n_{1}=0, n_{2}=1}^{\infty}\left[\left(\omega / \omega_{0}\right)^{2}-\tilde{n}_{1}^{2}\right]\left[\left(\omega / \omega_{0}\right)^{2}-\tilde{n}^{2}\right]^{-1 / 2} \theta\left[\left(\omega / \omega_{0}\right)^{2}-\tilde{n}^{2}\right], \\
& D_{E}^{y}(\omega) / V=\frac{1}{\omega\left(L_{1} L_{2}\right)^{3 / 2}} \sum_{n_{1}=1, n_{2}=0}^{\infty}\left[\left(\omega / \omega_{0}\right)^{2}-\tilde{n}_{2}{ }^{2}\right]\left[\left(\omega / \omega_{0}\right)^{2}-\tilde{n}^{2}\right]^{-1 / 2} \theta\left[\left(\omega / \omega_{0}\right)^{2}-\tilde{n}^{2}\right], \\
& D_{E}^{z}(\omega) / V=\frac{1}{\omega\left(L_{1} L_{2}\right)^{3 / 2}} \sum_{n_{1}=1, n_{2}=1}^{\infty} \tilde{n}^{2}\left[\left(\omega / \omega_{0}\right)^{2}-\tilde{n}^{2}\right]^{-1 / 2} \theta\left[\left(\omega / \omega_{0}\right)^{2}-\tilde{n}^{2}\right], \\
& D_{H}^{x}(\omega) / V=\frac{1}{\omega\left(L_{1} L_{2}\right)^{3 / 2}} \sum_{n_{1}=1, n_{2}=0}^{\infty}\left[\left(\omega / \omega_{0}\right)^{2}-\tilde{n}_{1}{ }^{2}\right]\left[\left(\omega / \omega_{0}\right)^{2}-\tilde{n}^{2}\right]^{-1 / 2} \theta\left[\left(\omega / \omega_{0}\right)^{2}-\tilde{n}^{2}\right], \\
& D_{H}^{y}(\omega) / V=\frac{1}{\omega\left(L_{1} L_{2}\right)^{3 / 2}} \sum_{n_{1}=0, n_{2}=1}^{\infty}\left[\left(\omega / \omega_{0}\right)^{2}-\tilde{n}_{2}{ }^{2}\right]\left[\left(\omega / \omega_{0}\right)^{2}-\tilde{n}^{2}\right]^{-1 / 2} \theta\left[\left(\omega / \omega_{0}\right)^{2}-\tilde{n}^{2}\right], \\
& D_{H}^{z}(\omega) / V=\frac{1}{\omega\left(L_{1} L_{2}\right)^{3 / 2}} \sum_{n_{1}=0, n_{2}=0}^{\infty} \tilde{n}^{2}\left[\left(\omega / \omega_{0}\right)^{2}-\tilde{n}^{2}\right]^{-1 / 2} \theta\left[\left(\omega / \omega_{0}\right)^{2}-\tilde{n}^{2}\right] .
\end{aligned}
$$

The summation range of the double series in (3.8)-(3.13) is different for the $\boldsymbol{E}$ - and the $\boldsymbol{H}$-field. Summing up (3.8)-(3.13) we get the total mode density:

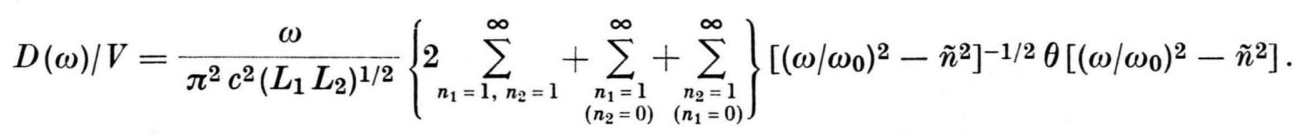

Equation(3.14)represents the eigenvalue spectrum of the EM-field in the rectangular wave guide. In a closed lossless system we have

$$
D(\omega)=\sum_{\{k\}} \delta\left(\omega-\omega_{k}\right) .
$$

Equation (3.15) is a series over the discrete modes of the system, $\omega=c|\boldsymbol{k}|$; the eigenvalues $\boldsymbol{k}$ are determined by the shape of the closed system. In the slab geometry - the distance of the perfectly conducting plates is $L-$ we find [13]:

$$
D(\omega) / V=\frac{\omega}{\pi c^{2} L}\left\{\frac{1}{2}+\sum_{n=1}^{\infty} \theta\left[\left(\frac{\omega}{\pi c / L}\right)^{2}-n^{2}\right]\right\} .
$$

Equation (3.14) is zero for $\omega<c \pi / \operatorname{Max}\left(L_{1}, L_{2}\right)$; for $\omega>c \pi / \operatorname{Max}\left(L_{1}, L_{2}\right)$ a continuous spectrum exists which tends to $+\infty$ at the points $\omega / \omega_{0}=\tilde{n}$ i.e. for $\tilde{n}(\lambda / 2)=\sqrt{L_{1} L_{2}} \quad(\lambda=$ wavelength $)$. The spectrum (3.16), however, has a continuous part beginning at zero frequency. With increasing $\omega$ successive finite jumps in $D(\omega)$ are observed at $n(\lambda / 2)=L$. Equation (3.16) can be considered as the superposition of a two dimensional continuous spectrum and a one dimensional discrete spectrum [24]. Similarly, Eq. (3.14) may be interpreted as the superposition of a discrete spectrum in two dimensions and a continuous spectrum in one dimension. As the corresponding EM-field is at least two dimensional, the spectrum (3.14) is only different from zero if also a discrete part is involved, i.e. if $\lambda / 2<\operatorname{Max}\left(L_{1}, L_{2}\right)$.

The representation of the partial mode densities in the form (3.6)-(3.13) is most useful for small frequencies because in this range only a few terms in the series have to be taken into account.

Consequently, for the calculation of the low temperature $\hbar \omega_{0}>k_{\mathrm{B}} T$ representation of the correlations (3.1) and (3.2) one should start from (3.6)-(3.13). The $\omega \rightarrow \infty$ representation, which is suitable for the calculation of the high temperature representation ( $\hbar \omega_{0} \ll k_{\mathrm{B}} T$ ) of the correlations, can be derived by applying the Poisson summation formula to (3.8)-(3.13). Introducing the quantity 


$$
\tilde{\boldsymbol{p}}=\left(\tilde{p}_{1}, \tilde{p}_{2}\right)=\left(\left(L_{1} / L_{2}\right)^{1 / 2} p_{1},\left(L_{2} / L_{1}\right)^{1 / 2} p_{2}\right), \quad|\tilde{\boldsymbol{p}}|=\tilde{p},
$$

we find:

$$
\begin{aligned}
& D_{\left\{{ }_{[E}\right\}}^{x}(\omega) / V=\frac{\omega^{2}}{3 \pi^{2} c^{3}}\left\{1+\frac{3}{2} \sum_{p_{1}, p_{2}=-\infty}^{\infty}\left[\frac{2 \sin \left(2 \pi \tilde{p} \omega / \omega_{0}\right)}{\left(2 \pi \tilde{p} \omega / \omega_{0}\right)^{3}}-\frac{2 \cos \left(2 \pi \tilde{p} \omega / \omega_{0}\right)}{\left(2 \pi \tilde{p} \omega / \omega_{0}\right)^{2}}\right]\right\} \\
& \mp \frac{\omega}{8 \pi c^{2} L_{2}}\left\{1+\sum_{p_{1}=-\infty}^{\infty}, \frac{2 J_{1}\left(2 \pi \tilde{p}_{1} \omega / \omega_{0}\right)}{\left(2 \pi \tilde{p}_{1} \omega / \omega_{0}\right)}\right\} \pm \frac{\omega}{4 \pi c^{2} L_{1}}\left\{1+\sum_{p_{2}=-\infty}^{\infty} J_{0}\left(2 \pi \tilde{p}_{2} \omega / \omega_{0}\right)\right\}-\frac{1}{4 \pi c L_{1} L_{2}}, \\
& D_{\left\{{ }_{H\}}\right\}}^{y}(\omega) / V=\frac{\omega^{2}}{3 \pi^{2} c^{3}}\left\{1+\frac{3}{2} \sum_{p_{1}, p_{2}=-\infty}^{\infty}\left[\frac{2 \sin \left(2 \pi \tilde{p} \omega / \omega_{0}\right)}{\left(2 \pi \tilde{p} \omega / \omega_{0}\right)^{3}}-\frac{2 \cos \left(2 \pi \tilde{p} \omega / \omega_{0}\right)}{\left(2 \pi \tilde{p} \omega / \omega_{0}\right)^{2}}\right]\right\} \\
& \pm \frac{\omega}{4 \pi c^{2} L_{2}}\left\{1+\sum_{p_{1}=-\infty}^{\infty} J_{0}\left(2 \pi \tilde{p}_{1} \omega / \omega_{0}\right)\right\} \mp \frac{\omega}{8 \pi c^{2} L_{1}}\left\{1+\sum_{p_{2}=-\infty}^{\infty} \frac{2 J_{1}\left(2 \pi \tilde{p}_{2} \omega / \omega_{0}\right)}{\left(2 \pi \tilde{p}_{2} \omega / \omega_{0}\right)}\right\}-\frac{1}{4 \pi c L_{1} L_{2}}, \\
& D_{\{H\}}^{z}(\omega) / V=\frac{\omega^{2}}{3 \pi^{2} c^{3}}\left\{1+3 \sum_{p_{1}, p_{2}=-\infty}^{\infty}\left[\frac{\sin \left(2 \pi \tilde{p} \omega / \omega_{0}\right)}{\left(2 \pi \tilde{p} \omega / \omega_{0}\right)}+\frac{2 \cos \left(2 \pi \tilde{p} \omega / \omega_{0}\right)}{\left(2 \pi \tilde{p} \omega / \omega_{0}\right)^{2}}+\frac{2 \sin \left(2 \pi \tilde{p} \omega / \omega_{0}\right)}{\left(2 \pi \tilde{p} \omega / \omega_{0}\right)^{3}}\right]\right\} \\
& \mp \frac{\omega}{8 \pi c^{2} L_{2}}\left\{1+2 \sum_{p_{1}=-\infty}^{\infty}\left[J_{0}\left(2 \pi \tilde{p}_{1} \omega / \omega_{0}\right)-\frac{J_{1}\left(2 \pi \tilde{p}_{1} \omega / \omega_{0}\right)}{\left(2 \pi \tilde{p}_{1} \omega / \omega_{0}\right)}\right]\right\} \\
& \mp \frac{\omega}{8 \pi c^{2} L_{1}}\left\{1+2 \sum_{p_{2}=-\infty}^{\infty},\left[J_{0}\left(2 \pi \tilde{p}_{2} \omega / \omega_{0}\right)-\frac{J_{1}\left(2 \pi \tilde{p}_{2} \omega / \omega_{0}\right)}{\left(2 \pi \tilde{p}_{2} \omega / \omega_{0}\right)}\right]\right\} \text {. }
\end{aligned}
$$

The upper and lower signs in $(3.18)-(3.20)$ refer to the $\boldsymbol{E}$ - and $\boldsymbol{H}$-field, respectively. $J_{0}$ and $J_{1}$ denote the ordinary Bessel functions of order zero and one. The primes at the summation signs express that the $\tilde{\boldsymbol{p}}=0$ and $\tilde{p}_{i}=0$ terms have already been extracted out of the double and the single series, respectively. They represent the "smoothed" partial mode densities.

Summing up $(3.18)-(3.20)$, the single series cancel out and we obtain

$$
\begin{aligned}
D(\omega) / V= & \frac{\omega^{2}}{\pi^{2} c^{3}}+\frac{\omega^{2}}{\pi^{2} c^{3}} \\
& \cdot \sum_{p_{1}, p_{2}=-\infty}^{\infty} \frac{\sin \left(2 \pi \tilde{p} \omega / \omega_{0}\right)}{2 \pi \tilde{p} \omega / \omega_{0}}-\frac{1}{2 \pi c L_{1} L_{2}} .
\end{aligned}
$$

The first term in (3.21) is the density of states in the isotropic infinite space. In the smoothed total mode density there are no terms proportional to the surface of the system.

Equations (3.18)-(3.20) and (3.21) may be interpreted as multiple reflection expansions: $p_{1}$ and $p_{2}$ are the numbers of reflections (in the sense of classical trajectories) at the walls $x=L_{2}$ and $y=L_{1}$, respectively. In (3.21) the double series vanishes if $\sqrt{\left(p_{1} L_{1}\right)^{2}+\left(p_{2} L_{2}\right)^{2}}$ is a multiple of half of the wavelength (closed trajectories).

The general concept to calculate mode densities by multiple reflection expansions has been developed by Balian and Bloch [25-27]. The expressions (3.18)-(3.20) are similar to the multiple reflection expansions (i.e. $\omega \rightarrow \infty$ representations) of the mode densities in rectangular cavities $[5,11]$ and in the slab resonator [6].

\section{Correlation Functions}

\section{1. $\alpha \rightarrow \infty$ Representation}

We insert (3.8)-(3.13) in (3.3) and (3.4). We expand the Bose distribution in an exponential series and note the integrals [28]

$$
\begin{aligned}
& \int_{1}^{\infty}\left(y^{2}-1\right)^{-1 / 2} \exp (-\lambda y) \mathrm{d} y=K_{0}(\lambda) \quad \text { and } \\
& \int_{1}^{\infty} y^{2}\left(y^{2}-1\right)^{-1 / 2} \exp (-\lambda y) \mathrm{d} y \\
& =\frac{1}{\lambda} K_{1}(\lambda)+K_{0}(\lambda) .
\end{aligned}
$$

$K_{v}$ represents the modified Bessel function [29].

With the definitions

$$
\alpha=\hbar \omega_{0} /\left(k_{\mathrm{B}} T\right)
$$

and

$$
\varkappa=2\left(L_{1} L_{2}\right)^{3 / 2} /\left(V \pi^{2}\right)
$$


we find:

$$
\begin{aligned}
& \varkappa \Gamma_{E}^{x}(\tau) /\left(\hbar \omega_{0}\right)=\sum_{p=1}^{\infty} \sum_{n_{1}=0, n_{2}=1}^{\infty} \frac{4}{\pi} \tilde{n}^{2}\left\{\frac{K_{1}\left[\tilde{n}\left(\alpha p-i \omega_{0} \tau\right)\right]}{\tilde{n}\left(\alpha p-i \omega_{0} \tau\right)}+\left(\tilde{n}_{2}^{2} / \tilde{n}^{2}\right) K_{0}\left[\tilde{n}\left(\alpha p-i \omega_{0} \tau\right)\right]\right\}, \\
& x \Gamma_{E}^{y}(\tau) /\left(\hbar \omega_{0}\right)=\sum_{p=1}^{\infty} \sum_{n_{1}=1, n_{2}=0}^{\infty} \frac{4}{\pi} \tilde{n}^{2}\left\{\frac{K_{1}\left[\tilde{n}\left(\alpha p-i \omega_{0} \tau\right)\right]}{\tilde{n}\left(\alpha p-i \omega_{0} \tau\right)}+\left(\tilde{n}_{1}^{2} / \tilde{n}^{2}\right) K_{0}\left[\tilde{n}\left(\alpha p-i \omega_{0} \tau\right)\right]\right\}, \\
& \varkappa \Gamma_{E}^{z}(\tau) /\left(\hbar \omega_{0}\right)=\sum_{p=1}^{\infty} \sum_{n_{1}=1, n_{2}=1}^{\infty} \frac{4}{\pi} \tilde{n}^{2} K_{0}\left[\tilde{n}\left(\alpha p-i \omega_{0} \tau\right)\right], \\
& x \Gamma_{H}^{x}(\tau) /\left(\hbar \omega_{0}\right)=\sum_{p=1}^{\infty} \sum_{n_{1}=1, n_{2}=0}^{\infty} \frac{4}{\pi} \tilde{n}^{2}\left\{\frac{K_{1}\left[\tilde{n}\left(\alpha p-i \omega_{0} \tau\right)\right]}{\tilde{n}\left(\alpha p-i \omega_{0} \tau\right)}+\left(\tilde{n}_{2}{ }^{2} / \tilde{n}^{2}\right) K_{0}\left[\tilde{n}\left(\alpha p-i \omega_{0} \tau\right)\right]\right\}, \\
& x \Gamma_{H}^{y}(\tau) /\left(\hbar \omega_{0}\right)=\sum_{p=1}^{\infty} \sum_{n_{1}=0, n_{2}=1}^{\infty} \frac{4}{\pi} \tilde{n}^{2}\left\{\frac{K_{1}\left[\tilde{n}\left(\alpha p-i \omega_{0} \tau\right)\right]}{\tilde{n}\left(\alpha p-i \omega_{0} \tau\right)}+\left(\tilde{n}_{1}{ }^{2} / \tilde{n}^{2}\right) K_{0}\left[\tilde{n}\left(\alpha p-i \omega_{0} \tau\right)\right]\right\}, \\
& x \Gamma_{H}^{z}(\tau) /\left(\hbar \omega_{0}\right)=\sum_{p=1}^{\infty} \sum_{n_{1}=0, n_{2}=0}^{\infty} \frac{4}{\pi} \tilde{n}^{2} K_{0}\left[\tilde{n}\left(\alpha p-i \omega_{0} \tau\right)\right] .
\end{aligned}
$$

If $L_{1}=L_{2}$ the $x$ - and $y$-components of the correlations are equal. For large moduli of the arguments of the modified Bessel functions we may expand these functions [29].

The coherence properties of the radiation field are described by the complex degrees of coherence [30]. Due to the Gaussian character of thermal light it is sufficient to consider the second order degree of coherence.

We put $L_{1}=L_{2}$ and consider the limit $\alpha \gg 1$. We expand the modified Bessel functions and take into account only the largest term in the series (4.3) and (4.4). We obtain:

$\gamma_{E}^{x}=\Gamma_{E}^{x}(\tau) / \Gamma_{E}^{x}(0) \rightarrow e^{i \omega_{0} \tau}\left[1-i k_{\mathrm{B}} T \tau / \hbar\right]^{-1 / 2}$
$\gamma_{E}^{z}=\Gamma_{E}^{z}(\tau) / \Gamma_{E}^{z}(0) \rightarrow e^{i \sqrt{2} \omega_{0} \tau}\left[1-i k_{\mathrm{B}} T \tau / \hbar\right]^{-1 / 2}$

Both (4.9) and (4.10) describe a damped one mode behaviour. Equations (4.9) and (4.10) differ only be the different recurrance times: (4.9) reveals a complete cycle in the time necessary to go through $2 L$ while in (4.10) only the time $\tau=\sqrt{2} L / c$ is necessary.

In contrary to the slab resonator, where the anisotropy is very marked in the $\alpha \rightarrow \infty$ regime $[14,15]$, we find in the wave guide nearly identical behaviour for both components (4.9) and (4.10). The damping in (4.9) and (4.10) is a consequence of the single infinite dimension of the system. In the slab resonator with two infinite dimensions the damping factor in the component parallel to the plates has the form $\left(1-i k_{\mathrm{B}} T \tau / \hbar\right)^{-1}$ (see (34) in [14]).

\section{2. $\alpha \rightarrow 0$ Representation}

We insert (3.17)-(3.19) in (3.3) and (3.4), expand the Bose distribution in an exponential series and note the integral [28]

$$
\int_{0}^{\infty} e^{-a x} J_{v}(\beta x) \mathrm{d} x=\frac{\beta^{-v}\left[\left(\alpha^{2}-\beta^{2}\right)^{1 / 2}-\alpha\right]^{v}}{\left(\alpha^{2}+\beta^{2}\right)^{1 / 2}} .
$$

We find:

$$
\begin{aligned}
\varkappa \Gamma_{\left\{\begin{array}{l}
E \\
H
\end{array}\right\}}^{x}(\tau) /\left(\hbar \omega_{0}\right)= & 8 \sum_{n=1}^{\infty} \sum_{p_{1}, p_{2}=-\infty}^{\infty}\left[\left(n \alpha-i \omega_{0} \tau\right)^{2}+(2 \pi \tilde{p})^{2}\right]^{-2} \\
& \mp\left(L_{1} / L_{2}\right)^{1 / 2} \sum_{n=1}^{\infty} \sum_{p_{1}=-\infty}^{\infty}\left[\left(n \alpha-i \omega_{0} \tau\right)^{2}+\left(2 \pi \tilde{p}_{1}\right)^{2}\right]^{-3 / 2} \\
& \pm\left(L_{2} / L_{1}\right)^{1 / 2} \sum_{n=1}^{\infty} \sum_{p_{2}=-\infty}^{\infty} \frac{2\left(n \alpha-i \omega_{0} \tau\right)^{2}-\left(2 \pi \tilde{p}_{2}\right)^{2}}{\left[\left(n \alpha-i \omega_{0} \tau\right)^{2}+\left(2 \pi \tilde{p}_{2}\right)^{2}\right]^{5 / 2}}-\alpha^{-2} \zeta\left(2,1-i \omega_{0} \tau / \alpha\right)
\end{aligned}
$$




$$
\begin{aligned}
\varkappa \Gamma_{\left\{\begin{array}{l}
E \\
H
\end{array}\right\}}^{y}(\tau) /\left(\hbar \omega_{0}\right)= & 8 \sum_{n=1}^{\infty} \sum_{p_{1}, p_{2}=-\infty}^{\infty}\left[\left(n \alpha-i \omega_{0} \tau\right)^{2}+(2 \pi \tilde{p})^{2}\right]^{-2} \\
& \pm\left(L_{1} / L_{2}\right)^{1 / 2} \sum_{n=1}^{\infty} \sum_{p_{1}=-\infty}^{\infty} \frac{2\left(n \alpha-i \omega_{0} \tau\right)^{2}-\left(2 \pi \tilde{p}_{1}\right)^{2}}{\left[\left(n \alpha-i \omega_{0} \tau\right)^{2}+\left(2 \pi \tilde{p}_{1}\right)^{2}\right]^{5 / 2}} \\
& \mp\left(L_{2} / L_{1}\right)^{1 / 2} \sum_{n=1}^{\infty} \sum_{p_{2}=-\infty}^{\infty}\left[\left(n \alpha-i \omega_{0} \tau\right)^{2}+\left(2 \pi \tilde{p}_{2}\right)^{2}\right]^{-3 / 2}-\alpha^{-2} \zeta\left(2,1-i \omega_{0} \tau / \alpha\right) \\
x \Gamma_{\left\{\begin{array}{l}
E \\
H
\end{array}\right\}}^{z}(\tau) /\left(\hbar \omega_{0}\right)= & 8 \sum_{n=1}^{\infty} \sum_{p_{1}, p_{2}=-\infty}^{\infty}\left[\left(n \alpha-i \omega_{0} \tau\right)^{2}+(2 \pi \tilde{p})^{2}\right]^{-2} \\
& \pm\left(L_{1} / L_{2}\right)^{1 / 2} \frac{1}{2} \sum_{n=1}^{\infty} \sum_{p_{1}=-\infty}^{\infty} \frac{2\left(n \alpha-i \omega_{0} \tau\right)^{2}-\left(2 \pi \tilde{p}_{1}\right)^{2}}{\left[\left(n \alpha-i \omega_{0} \tau\right)^{2}+\left(2 \pi \tilde{p}_{1}\right)^{2}\right]^{5 / 2}} \\
& \mp\left(L_{2} / L_{1}\right)^{1 / 2} \sum_{n=1}^{\infty} \sum_{p_{2}=-\infty}^{\infty}\left[\left(n \alpha-i \omega_{0} \tau\right)^{2}+\left(2 \pi \tilde{p}_{2}\right)^{2}\right]^{-3 / 2}
\end{aligned}
$$

The upper and lower signs refer to the $\boldsymbol{E}$ - and the $\boldsymbol{H}$-correlations, respectively. $\zeta(a, s)$ denotes the generalized Riemann zeta function [31].

We have seen above that the Eqs. (4.3)-(4.8) can be considered as an expansion around the one mode limit. The $\tilde{p}=0$ terms in the threefold series of $(4.11)-(4.13)$ yield $8 / \alpha^{4} \zeta\left(4,1-i k_{B} T \tau / \hbar\right)$. In the limit $\alpha \rightarrow 0$ these terms are the dominant ones:

$$
\Gamma(\tau) / V \rightarrow 4 \frac{\left(k_{B} T\right)^{4}}{\pi(\hbar c)^{3}} \zeta\left(4,1-i k_{B} T \tau / \hbar\right) .
$$

They represent the correlations of the infinite space and, consequently, (4.11)-(4.13) may be interpreted as the expansion around the isotropic space limit. The $\tilde{p}_{i}=0$ terms in the double series of (4.11)-(4.13) yield

$$
\pm\left(L_{i} / L_{j}\right)^{1 / 2} \alpha^{-3} \zeta\left(3,1-i k_{\mathrm{B}} T \tau / \hbar\right) .
$$

The $\tilde{\boldsymbol{p}}=0$ terms in $(4.11)-(4.13)$ result from the smoothed partial mode densities, i.e. from the

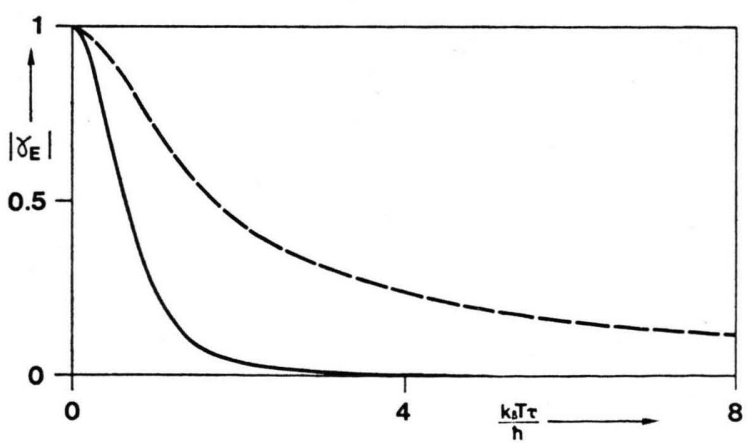

Fig. 1. Modulus of the temporal degree of coherence for the rectangular wave guide as a function of the thermodynamic reduced time $k_{\mathrm{B}} T \tau / \hbar$ for $\alpha \rightarrow \infty$ (dashed line) i.e. one mode limit and for $\alpha \rightarrow 0$ (solid line) i.e. the thermodynamic limit. $\tilde{\boldsymbol{p}}=0$ terms in $(3.18)-(3.20)$. Corresponding expansions in the case of the cube and the slab resonator are equally well known $[5,6]$.

In Fig. 1 we have plotted the modulus of $\gamma_{E}^{i}$ for both limits $\alpha \rightarrow \infty$ and $\alpha \rightarrow 0$, i.e. Eq. (4.14) and the first term in the normalized correlations $(4.17)-(4.19)$. The modulus of the (second) degree of coherence for $0<\alpha<\infty$ lies between these two limiting curves.

As we have already mentioned, in contrast to the slab resonator there is no remarkable anisotropy (see e.g. Fig. 3 in [14]).

In the wave guide closed trajectories are possible $-c \tau=2\left[\left(L_{1} p_{1}\right)^{2}+\left(L_{2} p_{2}\right)^{2}\right]^{1 / 2}-$ and therefore, as in the slab resonator, the phases of $\gamma$ reveal complete cycles while in the infinite isotropic space the phases of $\gamma$ tend to the value $\frac{3}{2} \pi[6,14]$.

\section{Internal Energy}

With the aid of (3.5) we can write

$E(T)=\int_{0}^{\infty} \hbar \omega\left[\exp \left(\hbar \omega / k_{\mathrm{B}} T\right)-1\right]^{-1} D(\omega) \mathrm{d} \omega$.

Inserting (3.14) in (5.1) we get the $\alpha \rightarrow \infty$ representation of the internal energy:

$$
\begin{aligned}
\frac{\pi \varkappa}{2 \hbar \omega_{0}} E(T)= & \pi^{-1} \sum_{p=1}^{\infty}\left\{2 \sum_{n_{1}, n_{2}=1}^{\infty}+\sum_{n_{1}=1}^{\infty}+\sum_{n_{2}=1}^{\infty}\right\} \\
& \cdot \tilde{n}^{2}\left[K_{1}(\alpha \tilde{n} p) /(\alpha \tilde{n} p)+K_{0}(\alpha \tilde{n} p)\right] .
\end{aligned}
$$

In the limit $\alpha \rightarrow \infty, E(T)$ shows the exponential behaviour (we put $L_{1}=L_{2}=L$ ):

$$
E(T) \sim \alpha^{-1 / 2} \exp (-\alpha) .
$$




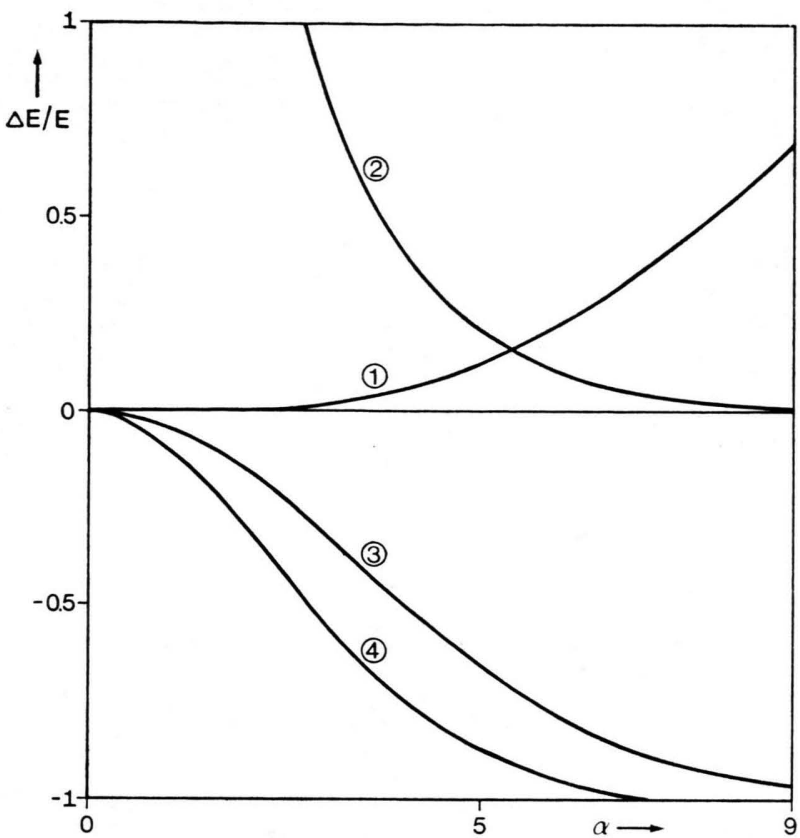

Fig. 2. Relative deviation of the radiation energy in the slab resonator (1), in the cube (4) and in the wave guide (3) from the Stefan Boltzmann law:

$$
\Delta E / E=\frac{\pi \varkappa \alpha^{4}}{12 \zeta(4) \hbar \omega_{0}} E(T)-1
$$

The relative deviation of the radiation energy in the slab resonator from the $T^{3}$ law is also displayed (2):

$$
\Delta E / E=\frac{\pi \varkappa \alpha^{3}}{2 \zeta(3) \hbar \omega_{0}} E(T)-1 .
$$

[1] L. S. Brown and G. J. Maclay, Phys. Rev. 184, 1272 (1969).

[2] K. M. Case and S. C. Chiu, Phys. Rev. A 1, 1170 (1970).

[3] H. P. Baltes, Appl. Phys. 1, 39 (1973).

[4] H. P. Baltes, E. R. Hilf, and M. Pabst, Appl. Phys. 3, 21 (1974); Appl. Phys. 5, 83 (1974).

[5] B. Steinle, H. P. Baltes, and M. Pabst, Phys. Rev. A 12,1519 (1975).

[6] H. P. Baltes, B. Steinle, and M. Pabst, Phys. Rev. A 13, 1866 (1976).

[7] G. S. Agarwal, Phys. Rev. A 11, 230 (1975).

[8] G. S. Agarwal, Phys. Rev. A 11, 243 (1975).

[9] G. S. Agarwal, Phys. Rev. A 11, 253 (1975).

[10] G. S. Agarwal, Phys. Rev. A 12, 1475 (1975).

[11] W. Eckhardt, Z. Phys. B 23, 213 (1976).

[12] W. Eckhardt, Z. Phys. B 26, 291 (1977).

[13] W. Eckhardt, Phys. Lett 62 A, 386 (1977).

[14] W. Eckhardt, Phys. Rev. A 17, 1093 (1978).

[15] W. Eckhardt, Z. Phys. B 31, 217 (1978).

[16] H. P. Baltes, Appl. Phys. 12, 221 (1977) (Review).

[17] R. C. Bourett, Nuovo Cim. 18, 347 (1960).

[18] J. Sarfatt, Nuovo Cim. 28, 401 (1963).
Therefore, $E(T)$ cannot be expanded in a Taylor series around $\alpha=\infty$, resp. $T=0$.

In the slab geometry the internal energy reveals an $\alpha^{3}$-law for $\alpha \rightarrow \infty$ [13]. In the cube of edge length $L$ also an exponential law is valid:

$$
E(T) \sim \exp (-\sqrt{2} \alpha) \quad[3] .
$$

We always expect an exponential law for $\alpha \rightarrow \infty$ if the eigenvalue spectrum is empty between $\omega=0$ and $\omega=\omega_{\min }>0$.

The $\alpha \rightarrow 0$ representation yields

$$
\begin{aligned}
\frac{\pi \varkappa}{2 \hbar \omega_{0}} E(T)= & \frac{\pi^{4}}{15 \alpha^{4}}-\frac{\pi}{12 \alpha^{2}}+\sum_{p_{1}, p_{2}=-\infty}^{\infty}, \\
& \cdot\left\{\frac{1}{16 \pi^{4} \tilde{p}^{4}}-\frac{\pi^{2} \cosh \left(\frac{2 \pi^{2}}{\alpha} \tilde{p}\right)}{2 \alpha^{3} \tilde{p}\left(\sinh \frac{2 \pi^{2}}{\alpha} \tilde{p}\right)^{3}}\right\} .
\end{aligned}
$$

In Fig. 2 we have plotted the relative deviations of the internal energy in the cube $\left(V=L^{3}\right)$ [3], in the slab geometry (distance $L$ ) [13] and in the wave guide $\left(L_{1}=L_{2}=L\right)$ from the $T^{4}$ law which is valid in the isotropic infinite space. In addition we displayed the relative deviation of the internal energy in the slab resonator from the $T^{3}$ law [13].

We note that the volume averaged energy density in the cube as well as in the wave guide lies below, while the energy density in the slab resonator lies above the infinite isotropic space limit.

[19] C. L. Mehta and E. Wolf, Phys. Rev. 134, A 1143 (1964).

[20] C. L. Mehta and E. Wolf, Phys. Rev. 134, A 1149 (1964).

[21] C. L. Mehta and E. Wolf, Phys. Rev. 161, 1328 (1967).

[22] L. D. Landau and E. M. Lifschitz, Elektrodynamik der Kontinua 2nd ed. Akademie Verlag, Berlin 1971, pp. $420-428$.

[23] R. Kubo, Rep. Progr. Phys. 29, 255 (1966).

[24] W. Eckhardt, Z. Naturforsch. 32a, 1184 (1977).

[25] R. Balian and C. Bloch, Ann. Phys. 60, 401 (1970).

[26] R. Balian and C. Bloch, Ann. Phys. 64, 271 (1971).

[27] R. Balian and C. Bloch, Ann. Phys. 69, 76 (1972).

[28] I. S. Gradshteyn and M. Ryzhik, Table of Integrals, Series and Products, 4th ed., Academic Press, New York 1965.

[29] M. Abramowitz and I. Stegun, Handbook of Mathematical Tables and Functions, Dover, New York 1965.

[30] L. Mandel and E. Wolf, Rev. Mod. Phys. 37, 231 (1965).

[31] A. Erdélyi, Higher Transcendental Functions Vol. 1, McGraw-Hill, New York 1953. 